Cognitive Behavioral Therapy (CBT) and Meditation Awareness Training (MAT) for the Treatment of Co-occurring Schizophrenia and Pathological Gambling:

A Case Study 


\begin{abstract}
There is a paucity of interventional approaches that are sensitive to the complex needs of individuals with co-occurring schizophrenia and pathological gambling. Utilizing a singleparticipant design, this study conducted the first clinical evaluation of a novel and integrated non-pharmacological treatment for a participant with dual-diagnosis schizophrenia and pathological gambling. The participant underwent a 20-week treatment course comprising: (i) an initial phase of second-wave cognitive behavioral therapy (CBT), and (ii) a subsequent phase employing a meditation-based recovery model (involving the administering of an intervention known as Meditation Awareness Training). The primary outcome was diagnostic change (based on DSM-IV-TR criteria) for schizophrenia and pathological gambling. Secondary outcomes were: (i) psychiatric symptom severity, (ii) pathological gambling symptom severity, (iii) psychosocial functioning, and (iv) dispositional mindfulness. Findings demonstrated that the participant was successfully treated for both schizophrenia and pathological gambling. Significant improvements were also observed across all other outcome variables and positive outcomes were maintained at three-month follow-up. An initial phase of CBT to improve social coping skills and environmental mastery, followed by a phase of meditation-based therapy to increase perceptual distance from mental urges and intrusive thoughts, may be a diagnostically-syntonic treatment for co-occurring schizophrenia and pathological gambling.
\end{abstract}

Key Words: Cognitive Behavioral Therapy, Meditation Awareness Training, Schizophrenia, Pathological Gambling, Mindfulness, Meditation 


\section{Cognitive Behavioral Therapy (CBT) and Meditation Awareness Training (MAT) for the Treatment of Co-occurring Schizophrenia and Pathological Gambling: \\ A Case Study}

\section{Introduction}

Approximately $10-18 \%$ of individuals with schizophrenia exhibit maladaptive gambling behavior (Cunningham-Williams, Cottler, Compton, \& Spitznagel, 1998; Desai \& Potenza, 2009). This compares with a prevalence estimate of less than $1 \%$ (UK) to $2.5 \%$ (US and Canada) for problem gambling in the general population (Chou \& Afifi, 2011; Wardle et al., 2011). According to Echeburúaa, Gómezc, and Freixac (2011), there exists a negativelyperpetuating bi-directional relationship between schizophrenia and problem/pathological gambling because the positive symptoms of schizophrenia (e.g., hallucinations, delusions, intrusive thoughts, etc.) tend to obscure risk perception. In turn, obscured risk perception (i.e., (judgement bias) increases the risk of gambling-related losses/debts and associated anxietyinduced acute psychotic episodes (Borras \& Haguelet, 2007). Furthermore, dysphoric mood states associated with schizophrenia tend to fuel addictive behaviors due to the individual engaging maladaptive avoidance strategies (Desai \& Potenza, 2009). In fact, individuals with a dual diagnosis of schizophrenia and pathological gambling are more likely to utilize outpatient services, derive reduced gains from dedicated schizophrenia treatments, and are at increased risk for relapse or premature therapy termination (Echeburúaa et al., 2011).

Despite the higher incidence rates of problem and pathological gambling in schizophrenia populations, individuals with a dual-diagnosis of schizophrenia and pathological gambling represent an under-researched population. To date, the results of only three treatment studies have been published. The first two of these were clinical case studies that employed serotonin/dopamine antagonist treatment with adjunctive cognitive behavioral therapy (CBT) 
(i.e., Borras \& Huguelet, 2007; Potenza \& Chambers, 2001). The third study utilized a smallsample pilot design ( $n=23$ intervention participants; $n=21$ control participants $)$ and assessed whether participants responded better to CBT plus treatment as usual (TAU: consisting of antipsychotics and mood stabilizers) versus TAU (Echeburúaa et al., 2011). Although each of these studies reported favourable outcomes at the post-intervention assessment stage, a follow-up assessment to determine maintenance effects was either not conducted, or demonstrated that participants had relapsed to pre-intervention levels of gambling involvement.

The CBT modality employed in these three studies belongs to the 'second wave' of CBT approaches. Second-wave CBT advocates the intentional controlling and modification of maladaptive cognitions (Wells, 1997), and is recommended for the treatment of both singlediagnosis schizophrenia and pathological gambling (National Institute for Health and Clinical Excellence [NICE], 2009; Rickwood, Blaszczynski, Delfabbro, Dowling, \& Heading, 2010). Despite this, second-wave CBT is not an acceptable treatment for all schizophrenia or pathological gambling patients. Indeed, relapse and rehospitilization rates following treatment by CBT can be as high as 75\% (Hodgins, Currie, el-Guebaly, \& Diskin, 2007; Jones, Hacker, Cormac, Meaden, \& Irving, 2013). Thus, there is a need for dedicated and novel interventional approaches that are sensitive to the complex range of factors that underlie the co-occurring schizophrenia and pathological gambling condition.

To overcome some of the limitations of second-wave CBT approaches and in order to offer alternative treatments to service-users, over the course of the last two decades, a 'third-wave' of CBT interventions have been formulated and clinically deployed. Unlike second-wave CBT, third-wave CBT techniques operate via a mechanism of transformative meditative awareness and perceptual re-distancing (Shonin, Van Gordon, Slade, \& Griffiths, 2013). 
Third-wave CBT techniques have their routes in Buddhist practice and involve training patients to accept and become fully aware of present moment events and experience.

Preliminary findings indicate that third-wave CBT approaches may be effective treatments for problem/pathological gambling (see reviews by: de Lisle, Dowling, \& Allen, 2011; Shonin, Van Gordon, \& Griffiths, 2013a). However, the case for utilizing third-wave techniques as schizophrenia interventions is less clear. Indeed, although there is some standalone evidence for the utility of third-wave CBT techniques as schizophrenia treatments (e.g., Brown, Davis, LaRoccod, \& Strasburger, 2010), due to the inherently subtle nature of meditation practice, caution is advocated prior to exposing people with schizophrenia to meditative training (Shonin, Van Gordon, \& Griffiths, 2013b). Furthermore, vis-à-vis secondwave CBT and as a standalone practice, meditation does not entail the same range of techniques (e.g., role plays, exposure exercises, life skill acquisition, assertiveness training, goal setting, etc.) that are specifically designed to enhance social and/or environmental coping skills.

Symptoms such as thought rumination, fantasizing, dissociation from the present moment, and reward-directed behavioral disturbance are common to both schizophrenia (Potenza \& Chambers, 2001; Renard, Pijnenborg, \& Lysaker, 2012) and pathological gambling (e.g., Griffiths, Wood, Parke, \& Parke, 2006). Given that meditation has been shown to reduce these symptoms (Shonin, Van Gordon, \& Griffiths, 2013c), it is hypothesized that meditation-based therapy (i.e., based on third-wave CBT principles) could be an acceptable and effective treatment for patients with co-morbid schizophrenia and pathological gambling. To overcome concerns regarding the use of meditation for people with schizophrenia, it is also hypothesized that such a phase of meditative training could lead to lasting therapeutic gains if administered following an initial phase of second-wave CBT (i.e., that focused on 
augmenting social coping skills, environmental mastery, and resilience to psychotic episodes). Thus, the purpose of this study is to conduct the first single-case assessment of a treatment combining phases of both second-wave and third-wave CBT for a patient with dual diagnosis schizophrenia and pathological gambling.

\section{Case Vignette and Assessment}

\section{Clinical history}

"Maria" (a pseudonym) is a 32-year old single white female without dependants, and with a history of moderate depressive episodes that lasted for a period of approximately 12 months (ending in early 2011). In mid-2011, Maria experienced several acute psychotic episodes and was subsequently diagnosed with Schizophrenia, Paranoid Type, Episodic with no Interepisode Residual Symptoms, With Prominent Negative Symptoms (DSM-IV-TR code 295.30). In early-2012, Maria began to exhibit pathological gambling behavior (mostly related to online slot-machine gambling) and was thus further diagnosed with Pathological Gambling (DSM-IV-TR code 312.31).

Maria's medical history showed no prior episode or complaint of mental illness beyond the most recent 42 months. For the first year of this 42 month period, Maria had followed a prescription of orally administered anti-depressants (paroxetine) that appeared to reduce her depressive symptoms to normative levels of severity. However, since the onset of psychotic symptoms (i.e., in mid-2011), Maria refused further pharmacotherapy. In early 2012, Maria commenced a course of CBT (second-wave) but missed several appointments and discontinued the therapy after nine weeks. 


\section{Case history}

Occupational history: Maria was employed as an ante-natal nurse and had worked at several hospitals. She had been on long-term sick leave since mid-2011 but had not actually worked since the beginning of 2011. This was because Maria's sick leave immediately followed a six-month period where she was suspended from her post due to being implicated in an alleged case of professional misconduct (involving the death of a new-born baby). Whilst the investigation cleared Maria of any negligence, it left her feeling angry, betrayed, and totally disillusioned after having dedicated more than ten years of her life to the nursing profession. Maria reported that although formal proceedings were now closed, she felt estranged from many of her former colleagues whom she stated had "distanced themselves" during the investigation.

Family history: Maria was raised by her biological mother and father who were married and are now approaching state retirement age. Maria's mother worked part-time as an office receptionist and Maria's father was employed fulltime as a skilled labourer. Maria stated that her parents showed a great deal of concern and were supporting her emotionally. She reported that she was close to both parents and "could not ask for anything more". Maria had a younger brother who worked for a mobile phone operator in London and visited her regularly. Maria found such visits to be uplifting.

Educational history: Maria's education was by way of state schooling where she achieved average grades. After completion of her general exams at a basic level (i.e., General School Certificates of Education), Maria completed a vocational qualification followed by further training to become a nurse. 
Social history: Maria had been socially active for the major part of her adult life. In her mid20 s, she enjoyed being on the steering committee of a local swimming club and up until 2011, she met with friends on a biweekly basis. However, since then, Maria had gradually withdrawn socially. She explained that this was because she felt "frightened that people might recognize me and know that I was suspended from work". Maria used to enjoy a regular routine of attending fitness classes but had not attended any in the 12 months prior to treatment. She had been involved in several short-term (i.e., 3-9 months) relationships with various male partners with the most recent relationship ending in 2010.

\section{Behavioral observations}

At her initial assessment, Maria seemed to be slightly overweight. Her appearance was unkempt and her hair appeared to be unwashed and unbrushed. Whilst not overtly noticeable, upon closer inspection it was apparent that Maria's jumper had been put on inside out. Maria's communication seemed to shift from phases of communicating in a forthcoming and coherent manner, to phases of mildly incoherent and lethargic speech that would often follow a long delay in responding. During such phases (which generally lasted for no more than five minutes), Maria would often respond verbally in an emotionless and expressionless manner whilst using a monotonous voice tone.

At the start of the initial assessment, Maria seemed to be cognizant as to person, place, time, and circumstance. However, as the consultation progressed, Maria's alertness deteriorated and she began to exhibit signs of disassociation. Maria experienced three episodes of heavy crying during her initial assessment although she could not identify what had triggered them. Verbal assertions such as "I know I'm useless", "I'm losing my mind", and "I'm never going to get back on my feet" were expressed. Maria appeared to be agitated and stated that she felt "exhausted" but at the same time found it "impossible to relax". 
Maria appeared to struggle to remember details about her activities during the few days immediately prior to the initial assessment. She was administered a three-object recall test but indicated no impairment in short-term memory functioning (this was consistent with findings from a recent neuropsychological examination that had ruled-out any neurodegenerative impairment). Maria showed a lack of interest in world affairs and towards events happening in her family life. For example, when talking of the recent birth of her nephew, she stated that babies are born all the time and "I don't see what everybody is so happy about".

\section{Presenting complaints}

Maria stated that she had experienced several episodes of moderately intense auditory hallucinations with the most recent episode occurring three months prior to the initial assessment. This most recent episode occurred whilst Maria was engaging in her normal thought rumination concerning the situation at work when she later realized that unfamiliar voices had been in the conversation. Maria confirmed that the voices were distinct from her own thoughts and had not occurred during hypnagogic or hypnopompic phases. Maria described the voices as worrisome and "annoying" but did not find them to be excessively persecutory. She indicated that the hallucinations did not seem to significantly impair her general levels of awareness or cognitive functioning. Maria denied any further episode of memorable hallucination, delusion, or flashback, but admitted prolonged and excessive daydreaming and thought rumination. She also denied any homicidal or suicidal ideations and denied any substance or alcohol dependency.

Maria stated that she frequently experienced difficulty sleeping. She recounted that when trying to go to sleep, she would often see visions of online slot machines, and/or would fantasize about a jackpot win. Maria implied that her sleeping difficulties were also due to worrying about her financial situation and attributed this worry as the cause of recurring 
migraines. Maria explained that over the course of the last six months, the average amount of time spent gambling at online sites had increased from 20 minutes to three hours per day. Maria’s typical gambling spend was in the region of $£ 200$ every week. However, Maria admitted that on several occasions she had spent up to $£ 125$ in a single two-hour session whilst trying to recoup gambling losses. Maria disclosed that her parents were assisting with paying off gambling-related debts but changed the subject whenever she was asked about the extent of the debts. Maria stated that during the previous year she had managed to refrain from gambling on three separate occasions with each abstinence period typically lasting ten days. Maria also stated that although her gambling activities were a major cause of anxiety, her hallucinatory episodes and overall levels of psychological distress seemed to intensify during phases of gambling abstinence.

\section{Diagnostic impression}

Maria exhibited a mild-to-moderate degree of disorganized behavior (such as frequent crying and confusion as to dress sense) coupled with chronic negative schizophrenia symptoms (e.g., apathy, avolition, anhedonia, flat affect, etc.) that occurred in conjunction with several hallucinatory episodes. However, Maria's current clinical picture was without prominent positive symptoms and therefore merited an anchoring diagnostic impression of Schizophrenia, Residual Type, With Prominent Negative Symptoms (DSM-IV-TR 295.6). A diagnosis impression of Mood Disorder with Psychotic Features (295.2) was discounted because Maria's psychotic episodes had occurred outside phases of exacerbated mood disturbance. Maria met more than five of the DSM-IV-TR maladaptive gambling behaviour criteria suggesting that her previous diagnosis of Pathological Gambling (212.31) was still current. 
The 18-item Brief Psychiatric Rating Scale (BPRS) (Overall \& Gorham, 1962) was administered to assess changes in the severity of psychiatric symptoms. Items on the BPRS are rated on a seven-point Likert scale $(1=$ not present, $7=$ extremely severe $)$ and responses are based on the previous seven-day period. Global scores range from 18-126. A global score of 31 corresponds to a 'mild' level of psychiatric illness, a score of 41 corresponds to 'moderately ill', and a score of 53 corresponds 'markedly ill' (Leucht et al., 2005). Maria's score at baseline was 57 .

The 12-item Gambling Symptom Assessment Scale (G-SAS) (Won Kim, Grant, Potenza, Blanco, \& Hollander, 2009) was used to assess changes in pathological gambling symptom severity. Items on the G-SAS are rated using a five-point Likert scale $(0=$ none, $4=$ extreme $)$ and responses are based on the previous seven-day period. Total scores range between 0 (no symptoms) and 48 (extreme) and G-SAS scores correspond to the following levels of symptom severity: 8-20 = mild, $21-30=$ moderate, $31-40=$ severe, $>41=$ extreme. Maria's baseline score was 40 .

The Global Assessment of Functioning Scale (GAF) (American Psychiatric Association, 2000) was used to assess changes in psychosocial functioning. Scores on the GAF range between 0-100 (higher scores reflect superior levels of functioning) and are subdivided into ten ranges of functioning. Working from the top level downwards, the decile that best reflects the individuals symptom severity or functioning is identified and a specific score (i.e., that falls within the 10-point range) is then determined. Maria's GAF score on intake was 40 (indicating impairment in reality testing and communication or major impairment in areas such as work, family relations, judgment, thinking, and/or mood). 
The 15-item Mindful Attention and Awareness Scale (MAAS) (Brown \& Ryan, 2003) was used to determine changes in levels of dispositional mindfulness. Scoring is frequency-based and comprises a six-point Likert scale (ranging from 'almost always' to 'almost never'). Scores range from 15 to 90 with higher scores reflecting greater degrees of dispositional mindfulness. Maria's baseline score was 38 .

Changes in gambling frequency, duration, and spend were assessed using daily dairy keeping by Maria. Maria's baseline scores (based on the preceding seven-day period) were as follows: gambling spend $=£ 210$ a week, gambling duration $=20$ hours a week, gambling frequency $=$ 26 episodes a week.

The Goal Attainment Scale (GAS) (Kiresuk \& Sherman, 1968) was administered to assess treatment goal attainment. The GAS allows for a series of goals to be agreed between client and therapist. Behavioral descriptions of functioning are then used to determine the level of goal achievement. Scores range from -2 (regression) through 0 (expected outcome attained) to +2 (expected outcome exceeded) for each of the agreed goals. Scores for individual goals are totalled and then the GAS conversion key for equally weighted scales is used to calculate an overall score. In this study, five equally-weighted goals were formulated. A score of 50 indicates an expected level of goal achievement (higher scores indicate greater levels of goal achievement).

The aforementioned measures were administered at four separate time points: (i) baseline ( $t 1$ ), (ii) mid-treatment ( $t 2$ [week 10]), (iii) therapy termination ( $t 3$ [week 20]), and (iv) threemonth follow-up (t4). All of the above scales are well-established measures with excellent levels of reliability and validity. 


\section{Case Formulation}

Maria's assessment indicated distorted and negative perceptions of self and environment that were heavily influenced by faulty cognitive processing. Thus, following informed consent, Maria's treatment was conceptualized based upon Beck's (1995) cognitive-behavioral formulation. According to cognitive theory, the manner in which an individual interprets and responds to a particular situation or stimuli is heavily influenced by underlying core beliefs. Activating events give rise to automatic thoughts that, if negatively orientated, have the effect of reifying any underlying detrimental schemas and thus perpetuating maladaptive cognitive, emotional, and behavioral responses (Beck \& Emery, 1985). Faulty cognitive processing is thought to play a significant role in the etiology of both schizophrenia (Wilson, Diamond, \& Factor, 1990) and pathological gambling (Davey, 2008).

\section{Predisposing factors and core beliefs}

Prior to her 36-month history of psychiatric complaint, Maria's functioning was psychosocially adaptive. Maria's parents were financially comfortable and had provided her and her only sibling with a secure and supportive family environment. However, Maria's relatively 'sheltered' upbringing had prevented her from acquiring the coping skills to effectively manage being on the receiving end of a professional negligence complaint of such magnitude. Indeed, when talking about the situation at work, Maria stated that "things like this don't happen to me" and "the complaint came completely out of the blue".

Although not a high-achiever per se, Maria had become accustomed to a steady level of career progression and her job had become increasingly important to her. Maria believed that her "career was going well until it happened" and had formulated a core belief about herself that she was "somebody who doesn't fail". Whilst such underlying beliefs were likely the 
very same drivers that motivated her to take her job seriously, they became maladaptive when they gave rise to intermediate assumptions such as "if people think I have failed they will reject me" and "people who are successful do not have significant setbacks".

When suddenly implicated in professional negligence proceedings, these underlying schemas played a pivotal role in initiating an unremitting sequence of catastrophizing automatic thoughts such as "whatever happens now is going to ruin my career and my life" and "even if I'm cleared people will still think I did something wrong". Such negatively orientated thinking served to strengthen Maria's core construct that others perceiving her to have failed would render life pointless. She automatically assumed that the complaint would cause her work colleagues and friends to reject her. Even when Maria's peers offered their support (e.g., by extending an invitation to meet for lunch), Maria believed that "it's all pretence, they don't care really".

Consequently, Maria progressively withdrew from potentially protective interpersonal interactions. This probably left Maria's co-workers feeling unwelcome and resulted in a 'reciprocal interaction' (Beck, Rush, Shaw, \& Emery, 1979) that further reified Maria's belief that she had been rejected by her peers. This led to Maria becoming increasingly more withdrawn, and she began to avoid situations requiring any level of social aptitude. Over the course of several months, Maria's coping resources rapidly diminished and she began to feel "exhausted" and "easily stressed out".

As a result of diminishing personal resources, Maria began to feel worthless and experienced increasing difficulty in transmuting negative affective states. Maria fell into a 'depressive interlock' (Clark, Beck, \& Alford, 1999) and began to feel resentful towards her employer. For example, notwithstanding the fact that it was normal protocol in such a situation, the 
decision of Maria's employer to suspend her made Maria feel that "they treat me like I'm just a part that needs replacing" and "I've wasted the last ten years of my life for those clowns".

Maria's perspective on life became increasingly contracted and phases of thought rumination increased in frequency and duration. Coupled with the traumatizing effect of being accused of professional negligence, this chronic negatively-orientated thought rumination likely predisposed Maria to low intensity positive and negative schizophrenia symptoms. Maria's hallucinatory episodes typically followed periods of social interaction with a family member. The most intrusive hallucination occurring after Maria had finished a long telephone conversation with her mother during which she was informed of the birth of her brother's child. Sensory and emotional overload when coupled with pre-existent negative symptoms have been identified as a risk-marker for hallucinatory episodes (Hemsley, 2005).

The onset of psychotic symptoms and declining overall levels of psychological wellbeing prompted Maria to look for a means of escaping her psychological environment. Consequently, she developed an obsession for online slot machine playing which she found particularly attractive due to the absence of any requirement for social interaction. Maria became myopically focussed on gambling rewards and her capacity for self-control rapidly deteriorated. Financial problems swiftly followed, and attempts to refrain from gambling left Maria feeling irritable and disorientated.

Maria's almost routine-like gambling behavior appeared to help regulate the frequency and severity of hallucinatory episodes. However, this coping strategy was maladaptive and resulted in increased personal conflict (e.g., negative affective states, self-blame, impaired relaxation ability, etc.) and gambling using progressively larger amounts of money in order to support sensation-seeking behavior. Although Maria's gambling problem was ultimately maladaptive, it helped to convince Maria that by modifying her behavior, it was actually 
possible to regulate the occurrence of hallucinatory episodes. At this point Maria experienced a renewed motivation to recover which appeared to coincide with her entering the residual phase of schizophrenia.

Residual-type schizophrenia (RTS) is generally regarded as a transitory state between fullblown psychotic episodes and remission (Davey, 2008). However, without successful treatment, RTS can be continuously present for many years (American Psychiatric Association, 2000). During the residual period, reductions in psychosocial functioning, deficits in perceptual and memory skills, and encumbered verbal fluency are symptomatic impairments (e.g., Park \& Holzman, 1992; Riley, McGovern, Mockler, \& Doku, 2000; Slaghuis \& Curran, 2001). Maria was aware that she had reached a pivotal stage in which she could easily relapse to further and more severe psychotic episodes. Maria acknowledged "if I don't get my head together now, I know that things are going to get much worse".

\section{Protective and problematic factors}

Whilst Maria's failure-averse core belief was maladaptive, if directed towards the achievement of therapeutic outcomes it could avail itself as a protective feature. Furthermore, although Maria had been disillusioned by her work situation, she still demonstrated some desire to revive her career. Coupled with a general motivation to improve her overall levels of wellbeing, this would likely constitute a further protective factor.

Maria's parents supported her both emotionally and financially. The absence of any immediate financial pressure helped provide Maria with a stress-limited environment conducive to therapeutic recovery. However, in the longer term, this could undermine Maria's impetus to return to work and could therefore become an obstacle to recovery. 


\section{Intervention}

Consistent with NICE (2009) guidelines for the treatment of schizophrenia, Maria had previously commenced a six-month program of CBT. However, Maria missed several sessions and discontinued the treatment after approximately two months. When initially presenting for therapy, Maria had expressed a wish to try meditation, and more specifically, to receive an intervention known as Meditation Awareness Training (MAT) (that she had read about in the newspaper).

Given that Maria had already commenced a course of CBT, it was felt that a further period of second-wave CBT would help to consolidate Maria's understanding of cognitive and behavioral processes. Consistent with the aforementioned recommendations to exercise caution when using meditation to treat schizophrenia, an initial phase of second-wave CBT was likewise deemed to be prudent in order to reduce the risk of latent positive schizophrenia symptoms resurfacing whilst Maria was undergoing meditative training. Furthermore, in light of Maria's tendency to not see commitments through to their fruition, it was felt that it would be beneficial for her to attempt to reach certain therapeutic milestones utilizing the therapeutic approach that she had formerly discontinued (i.e., second-wave CBT).

Thus, following informed consent, Maria received a 20-week course of treatment that was divided into two distinct stages: (i) an initial stage employing second-wave CBT principles (weeks 1-10), and (ii) a subsequent stage utilizing a meditation-based recovery model (weeks 11-20). Maria attended one session each week and each session lasted for either 50 minutes (weeks 1-10, 19 and 20) or 90 minutes (weeks 11-18). Sessions began with the setting of an agenda that was agreed between therapist and client, and all of the therapy was delivered by the first author (a senior psychotherapist and meditation teacher). 
The early interventional phase focussed on the establishment of therapeutic alliance that is known to play a particularly important role in augmenting outcomes from behavioral schizophrenia interventions (Frank \& Gunderson, 1990). Core therapeutic conditions such as active listening, unconditional positive regard, accurate empathy, reflective statements, respect, and genuineness were consistently employed (Wells, 1997). Self-disclosure was used to normalize situations and encourage discussion of sensitive topics. An example was the therapist sharing with Maria his mutual interest in swimming that helped to strengthen connection and make the issue of discussing Maria's non-attendance at her local health centre more approachable.

Psycheducation was important during this phase as a means of reinforcing Maria's understanding of the principles of $\mathrm{CBT}$ and to establish rationale behind goal formulation. Using real life examples, Ellis's (1970) ABC model was used to explain cognitive processing. Furthermore, a normalization destigmatization process was used to explain schizophrenia etiology, prevalence, and symptom course (Kingsom \& Turkington, 1994).

During the early treatment phase, five GAS compatible goals were agreed: (i) reduction of gambling spend to below $£ 30$ a week, (ii) returning to work on a part-time basis for 12-hours a week, (iii) developing social support systems, (iv) starting to drive again, and (v) reuptake of a regular exercise routine. During this stage, Maria was also introduced to Beck's (1984) Weekly Activity Schedule (WAS) as a way of addressing the disruption and loss of structure she had experienced whilst being absent from work. The WAS also used to enable Maria to identify possible environmental and behavioral stressors (i.e., activating events) as well as a means to help her understand her reactions to them. 
The mid-intervention phase centered upon the identification of distorted underlying beliefs and resultant automatic thoughts, as well as the implementation of reformative strategies. One of Maria's primary maladaptive core beliefs was that she had failed, and that this 'failure' could not be used as a catalyst for personal growth. Guidelines for the use of CBT for the treatment of schizophrenia recommend that rather than directly challenging assumptions that are not reality-based, that 'guided discovery', 'logic reasoning', and 'Socratic questioning' should be used to steer the client towards testing the validity of flawed assumptions (Turkington, Kingdon, \& Weiden, 2006).

Weekly collaborative review of the Dysfunctional Thought Diary (DTD) (Abramovich, 2006), that Maria was requested to complete on an ongoing basis, provides a good example of how these guidelines were implemented in the current study. During the earlyinterventional phase, Maria had received a letter from her employer inviting her to consider returning to work on a two-day per week basis (i.e., as a step-up to normal duties). However, due to the automatic thought "I cannot return to work because everybody thinks I'm incompetent", Maria chose not to respond. During a review of the DTD in the midinterventional phase, the therapist posed questions such as "How do you know everybody thinks that you are incompetent?" and "Is there any evidence that supports this view?" Following deliberation of these questions, Maria realised that cognitions such as " $m y$ employer does in fact want me to return", "in actual fact it is impossible for me to accurately know what other people are thinking", and "so far in my career I have actually been very competent" would have constituted a more proportionate and adaptive response.

Personalising and selective abstraction during social interactions were major sources of stress for Maria. The vulnerability-stress model (Zubin \& Spring, 1977) was used to help Maria 
comprehend her resilience deficit towards social stressors. Maria was then re-exposed to social interaction by way of various role plays in which a fellow therapist partook. One such role play included an assertiveness rehearsal exercise to build resilience in the eventuality of somebody inquiring about Maria's involvement in the negligence investigation.

An interest inventory was used to identify some of Maria's former hobbies and interests. Following several weeks of gentle encouragement, in the ninth week of the therapy Maria decided to recommence practicing the guitar and visited her local health centre to request a schedule of swimming-based fitness classes. As Maria's competence and range of activities slowly began to expand, 'mastery and pleasure ratings' (Bradshaw, 1998) were assigned to individual activities to determine proficiency and to identify any maladaptive thought processes that inhibited enjoyment. Behavioral experiments such as attending a creative arts class were likewise employed in order to build self-efficacy and to test Maria's assumptions that she was worthless and lacked creativity.

A key milestone in Maria's treatment (and indication that she was ready to progress to the meditative-phase of her treatment) was in the tenth week of therapy when she stated "although easier said than done, major set-backs happen to everybody and can actually help you learn more about yourself'.

Stage II (MAT: weeks 11-18)

Meditation Awareness Training (Van Gordon, Shonin, Sumich, Sundin, \& Griffiths, 2013; Shonin, Van Gordon, \& Griffiths, 2013d) is a secular intervention generally delivered over an eight-week period. Unlike existent mindfulness-based interventions, MAT follows a more comprehensive and traditional approach to meditation and incorporates practices that would be traditionally followed by Buddhist practitioners. These include (for example) techniques 
aimed at cultivating generosity, ethical awareness, patience, and compassion, as well as insight into subtle concepts such as impermanence.

Participants of MAT receive a program booklet and a CD comprising guided meditations in order to encourage them to introduce meditation practice into their normal daily routine. MAT is generally delivered as a group intervention and so Maria followed a modified version of the program. Key modifications included a reduction in the duration of individual sessions (from 120 to 90 minutes), and an integration of the one-to-one therapeutic component (normally administered as two 50-minute sessions in weeks 3 and 7 of the group program) into Maria's weekly 90-minute sessions. Each weekly session attended by Maria comprised three phases: (i) discussion with the therapist (approximately 35 minutes), (ii) a taught component (approximately 25 minutes), and (iii) a guided meditation (approximately 20 minutes). A short break (5-10 minutes) was scheduled immediately prior to the guided meditation.

Maria responded well to the meditative phase of treatment and commented that she enjoyed practicing meditation. Maria stated that meditation "makes me feel calmer, more caring, and more able to watch what the mind is getting up to". Maria also commented that meditation helped her to feel "more spiritual and at-home", and to understand that "thoughts and feelings have to dissolve - so I just relax the mind and don't hold on - then they start to go away on their own".

Stage II (therapy termination: weeks 19-20)

The late phase of treatment concentrated on preparing Maria for termination. Whilst Maria felt that her overall levels of psychological wellbeing had considerably improved, towards the end of the treatment she expressed concerns over relapse due to loss of therapeutic support. 
With such concerns in mind and in order to maximize the maintenance of any salutary outcomes, Maria was asked to continue with her daily practice of meditation and to keep a daily register of stress levels, sleep patterns, diet, and exercise. Following a review of treatment and progress, coping strategy cue cards were formulated that Maria agreed to refer to on a bi-weekly basis. Finally, a procedure for emergencies was discussed, dates and times for planned telephone contact were agreed, and three 50-minute booster sessions were arranged for Maria to attend at monthly intervals.

\section{Outcomes}

Maria attended a diagnostic interview (utilizing DSM-IV-TR diagnostic criteria) immediately following completion of the 20 -week therapeutic course (i.e., t3) and exhibited clinically significant levels of change (i.e., to below the diagnostic threshold) in schizophrenia and pathological gambling symptomatology. These diagnostic impressions remained unchanged at three-month follow-up (t4).

Maria's changes in psychometric test scores over time (i.e., the BPRS, GAF, G-SAS, \& MAAS) are shown in Figure 1. During the CBT phase of the therapy (i.e., $t 1-t 2$ ), Maria exhibited moderate levels of improvement in BPRS and GAF scores but no change in scores on the G-SAS or MAAS. However, at the end of the MAT treatment phase (i.e., t3), Maria demonstrated significant baseline-to-endpoint (i.e., $t 1-t 3$ ) improvements in all outcome variables. These improvements were maintained at follow-up, with a trend towards further improvements (i.e., $t 3-t 4)$ in levels of dispositional mindfulness (MAAS) and psychosocial functioning (GAF). Maria's $t 3$ and $t 4$ scores on the BPRS were within the normative range. Maria did not demonstrate any reductions in gambling spend, duration, or frequency during the CBT phase of the treatment. However, Maria achieved abstinence after completion of MAT and this was maintained at three month follow-up. 
At the end of the 20-week intervention, Maria had returned to work on a two-day a week (i.e., 16 hours) basis. Her post-treatment GAS score of 77 indicated achievement across all goal fronts, and was over and above the expected level. Maria had substantially increased her level of social engagement with peers, friends, and family members. She had resumed a routine of regular exercise and evinced greater self-efficacy in respect of work and household tasks. Maria's post-intervention (i.e., $t 3 \& t 4)$ GAF score of $>70$ indicated only a mild level of impairment in psychosocial functioning.

Maria attended every single one of the 20 weekly sessions and her completion rate on the DTD was $85 \%$. Since commencing MAT in the eleventh week, Maria had maintained a daily practice of meditation and her average daily practice time was 76 minutes. Maria adhered to a strict program of meditation practice consisting of four 20-minute meditation sessions a day: (i) when first awaking in the morning, (ii) at mid-day immediately after lunch, (iii) earlyevening before evening meal, and (iv) late evening just before going to bed.

[Insert Figure 1 around here]

\section{Discussion}

The purpose of this paper was to illustrate the case of an adult female ("Maria") with cooccurring schizophrenia and pathological gambling who was treated via an initial phase of second-wave CBT followed by a phase of MAT. Findings demonstrate that by the end of the 20-week treatment course, Maria no longer met DSM-IV-TR diagnostic criteria for either schizophrenia or pathological gambling. In addition to significant reductions in overall levels 
of psychiatric impairment and problem gambling, Maria exhibited significant improvements in levels of dispositional mindfulness, and psychosocial functioning. Positive outcomes were maintained at three-month follow-up.

Whilst Maria demonstrated a certain degree of improvement in psychiatric symptoms and psychosocial functioning during the initial 10-week CBT treatment phase, the greatest progress in her overall recovery was observed during the subsequent phase of meditationbased therapy. Interpreting these findings is not as simple as concluding that Maria responded more favourably to MAT than CBT as there are numerous factors that need to be considered. The initial CBT phase was incorporated into Maria's treatment in order to (i) establish therapeutic alliance, (ii) provide a grounded understanding of cognitive and emotional processes, (iii) improve social coping skills and environmental mastery, and (iv) reduce the risk of further hallucinatory episodes. Thus, although this preparatory treatment phase did not convert into significant therapeutic gains, it helped to improve Maria's functionality to a level where she would derive the maximum benefit from the inherently more subtle meditative approach. Of course, an alternative explanation of this finding is that having already commenced a program of CBT and not finished the therapeutic course, Maria was more motivated to try meditation as a novel alternative. However, this latter line of interpretation does not accord with qualitative feedback provided by Maria nor with the therapist's impression.

The relationship between schizophrenia and pathological gambling is complex and poorly understood, and people with this dual-diagnosis represent a unique psychiatric population with specific treatment needs. In general, the successful treatment of schizophrenia is considered to be contraindicated by maladaptive gambling behavior. However, Maria's case was distinctive in this respect because although detrimental in several other remits of 
functionality, Maria's gambling problem appeared to exert a protective influence over positive schizophrenia symptoms. To the authors' knowledge, this represents the first reported instance of this interaction which may have important implications for the treatment of people with comorbid schizophrenia and pathological gambling. For example, if the "daily structure" and cognitive involvement that result from pathological gambling serve to alleviate paranoid-type psychotic features, then consistent with the formulation of Maria's treatment in the current study, the focus of therapeutic efforts to reduce gambling involvement may be better reserved for the mid/latter phase of recovery (i.e., subsequent to an initial period targeting any prominent schizophrenia symptoms).

Maria demonstrated significant reductions in pathological gambling symptomatology during the latter phase of her treatment (i.e., whilst undergoing MAT). Recently, Shonin et al (2013a) proposed various mechanisms by which Buddhist-derived forms of meditation may ameliorate problem gambling severity. These include: (i) a greater capacity for meditative absorption leading to reductions in autonomic and psychological arousal, (ii) increased awareness of the impermanent nature of life and phenomena resulting in reductions in salience and myopic focus on reward (i.e., owing to better understanding that all that is won must ultimately be lost), (iii) increased levels of loving-kindness, compassion, and selfcompassion that facilitates the dismantling of maladaptive shameful and self-disparaging schemas, (iv) an undermining of the intrinsic value and 'authenticity' that problem gamblers assign to the gambling experience, (v) increased levels of meditative-born patience leading to reductions in impulsivity and frustration, and an improved capacity to defer gratification, and (vi) spiritual nourishment that attenuates feelings of loneliness and low sense of purpose.

An additional mechanism of action proposed in the same paper by Shonin et al (2013a) is that of 'bliss substitution'. According to Shonin et al, bliss and tranquillity are frequently reported 
outcomes of certain concentrative forms of Buddhist meditation. Based on the same premises that underlie the current use of substitution techniques in problem gambling treatments (e.g., see Jackson, Francis, Byrne, \& Christensen, 2012), bliss substitution may reduce the risk of relapse and temper withdrawal symptoms due to the individual developing a "positive addiction' towards meditation. Although in the current study 'addiction to meditation' was not explored as an outcome per se, Shonin et al's theory seems to be consistent with Maria's unremitting adherence to a strict routine of daily meditation practice.

Findings from the current study demonstrated that a multi-modal treatment formulation consisting of an initial phase of second-wave CBT followed by MAT led to the successful treatment of an adult female with dual-diagnosis schizophrenia and pathological gambling. The initial CBT phase focussed on reducing the risk of relapse to further hallucinatory episodes, and on targeting the negative symptoms of schizophrenia via the augmentation of social coping skills and personal resources. The subsequent phase of meditative-based therapy focussed on the development of a greater perceptual distance towards intrusive thoughts, emotions, and gambling urges. Based on qualitative feedback, the meditative phase also appeared to instil a greater sense of spiritual awareness, a compassionate regard for self and other, and insight into the impermanent nature of life and phenomena.

Whilst findings indicate that an initial period of second wave CBT followed by a phase of third-wave CBT may be an acceptable treatment for people with co-occurring schizophrenia and pathological gambling, they should be considered cautiously due to a number of limitations that are inherent to the case study design. Particularly noteworthy amongst these was the absence of a control condition and the fact that Maria's response to treatment may not be typical of all people with comorbid schizophrenia and pathological gambling. For example, Maria's motivation to recover, willingness to adhere to the treatment plan, and 
capacity/readiness to monitor and report symptom changes, are all predictors of a successful response to behavioral interventions (Foa \& Emmelkamp, 1983). People with more pronounced psychiatric symptoms and/or individuals who do not meet these favourable prognostic criteria may therefore respond less favourably to the challenges of this 20 -week multimodal treatment approach. Similarly, in addition to more conventional therapeutic techniques such as diary keeping, behavioural experiments, goal setting, and psychoeducation, the intervention employed a broad range of meditation exercises and meditation-based dialogue techniques that may exert specific training (and therefore time) demands on the part of the therapist. Other notable limitations were the absence of a longerterm follow-up period and the utilization of a preceding seven-day period for measuring gambling involvement (i.e., that does not control for short-term contextual factors such as availability of money). Thus, further clinical evaluation using both single-participant and controlled larger-sample designs is required in order to replicate these findings. 


\section{References}

Abramovich, E. (2006). Application of CBT in an inpatient setting. Case illustration of an adult male with anxiety, depression, Axis II symptoms. Clinical Case Studies, 5, 305330.

American Psychiatric Association (APA). (2000). Diagnostic and Statistical Manual of mental Disorders (4th ed., rev). Washington: APA.

Beck, A. (1984). Cognitive approaches to stress. In R. Woolfolk, \& P. Lehren. Principles and practice of stress management (pp. 125-155). New York: Guildford.

Beck, A., \& Emery, G. (1985). Anxiety disorders and phobias: A cognitive perspective. New York: Basic Books.

Beck, A., Rush, A., Shaw, B., \& Emery, G. (1979). Cognitive therapy of depression. New York: Guilford.

Beck, J. (1995). Cognitive therapy: Basics and beyond. New York: Guildford.

Borras, L., \& Huguelet, P. (2007). Schizophrenia and pathological gambling. American Journal on Addictions, 16, 269e271.

Bradshaw, W. (1998). Cognitive-behavioral treatment of schizophrenia: A case study. Journal of Cognitive Psychotherapy: An International Journal, 12, 13-25.

Brown, L. F., Davis, L. W., LaRoccod, V. A., \& Strasburger, A. (2010). Participant perspectives on mindfulness meditation training for anxiety in schizophrenia. American Journal of Psychiatric Rehabilitation, 13, 224-242. 
Brown, K. W., \& Ryan, R. M. (2003). The benefits of being present: Mindfulness and its role in psychological well-being. Journal of Personality and Social Psychology, 84, 822848.

Chou, K. L., \& Afifi, T. O. (2011). Disordered (pathological or problem) gambling and Axis I psychiatric disorders: Results from the National Epidemiologic Survey on alcohol and related conditions. American Journal of Epidemiology, 173, 1289-1297.

Clark, D., Beck, A., \& Alford, B. (1999). Scientific Foundations of Cognitive Theory and Therapy of Depression. New York: Wiley.

Cunningham-Williams, R., Cottler, L., Compton, W., Spitznagel, E. (1998). Taking chances: problem gamblers and mental health disorders. Results from the St. Louis Epidemiologic Catchment Area Study. American Journal of Public Health, 88, 10931096.

Davey, G. (2008). Psychopathology: Research, Assessment and Treatment in Clinical Psychology. Oxford: BPS Blackwell.

Desai, R. A., \& Potenza, M.N. (2009). A cross-sectional study of problem and pathological gambling in patients with schizophrenia/schizoaffective disorder. Journal of Clinical Psychiatry, 70, 1250-1257.

de Lisle, S. M., Dowling, N. A., \& Allen, J. S. (2011). Mindfulness and problem gambling: A review of the literature. Journal of Gambling Studies. doi:10.1007/s10899-011-92847. 
Echeburúaa, E., Gómezc , M., \& Freixac, M., (2011). Cognitive-behavioral treatment of pathological gambling in individuals with chronic schizophrenia: A pilot study. Behavior Research and Therapy, 49, 808-814.

Ellis, A. (1970). The essence of rational psychotherapy. A comprehensive approach to treatment. New York: Institute for Rational Living.

Foa, E., \& Emmelkamp, P. (1983). Failures in Behavior Therapy. New York: Wiley and Sons.

Frank, A., \& Gunderson, J. (1990). The role of the therapeutic alliance in the treatment of scihizophrenia: Relationship to course and outcome. Archives of General Psychiatry, 47, 228-236.

Griffiths, M. D. (2004). Betting your life on it: problem gambling has clear health related consequences. British Medical Journal, 329, 1055-1056.

Griffiths, M. D., Wood, R. A., Parke, J., \& Parke, A. (2006). Dissociative states in problem gambling. In C. Allcock (Ed.), Current issues related to dissociation (pp. 27-37). Melbourne: Australian Gaming Council.

Hemsley, D. (2005). The development of a cognitive model of schizophrenia: placing it in context. Neuroscience and Biobehavioral Reviews, 29, 977-988.

Hodgins, D. C., Currie, S. R., el-Guebaly, N., \& Diskin, K. (2007). Does providing extended relapse prevention bibliotherapy to problem gamblers improve outcome. Journal of Gambling Studies, 23, 41-54. 
Jackson, A. C., Francis, K. L., Byrne, G., \& Christensen, D. R. (2012). Leisure substitution and problem gambling: report of a proof of concept group intervention. International Journal of Mental Health and Addiction. doi:10.1007/s11469-012-9399-9.

Jones, C., Hacker, D., Cormac, I, Meaden, A., \& Irving, C. B. (2013). Cognitive behavior therapy versus other psychosocial treatments for schizophrenia. The Cochcrane Collaboration. doi: 10.1002/14651858.CD008712.pub2.

Kingsom, D., \& Turkington, D. (1994). Cognitive therapy of schizophrenia. New York: Guildford.

Kiresuk, T., \& Sherman, R. (1968). A reply to the critique of the goal attaining scaling. Social Work Research and Abstracts, 13, 9-11.

Leucht, S., Kane, J. M., Kissling, W., Hamann, J., Etschel, E., \& Engel, R. (2005). Clinical implications of Brief Psychiatric Rating scale scores. British Journal of Psychiatry, 187, 336-371.

National Institute for Health and Clinical Excellence (NICE). (2009). NICE Clinical Guideance 82: Schizophrenia - Core interventions in the treatment and management of schizophrenia in adults in primary and secondary care. Manchester: Author.

Overall, J. E., \& Gorham, D.R. (1962). The brief psychiatric rating scale. Psychological Reports, 10, 799-812.

Park, S., \& Holzman, P. (1992). Schizophrenics show spatial working memory deficits. Archives of General Psychiatry, 49, 975-982. 
Potenza, M. N., \& Chambers, R. A. (2001). Schizophrenia and pathological gambling. American Journal of Psychiatry, 158, 497e498.

Renard, S. B., Pijnenborg, M., \& Lysaker, P. H. (2012). Dissociation and social cognition in schizophrenia spectrum disorder. Schizophrenia Research, 137, 219-223.

Rickwood, D., Blaszczynski, A., Delfabbro, P., Dowling, N., \& Heading, K. (2010). The psychology of gambling. InPsych, 32, 11-21.

Riley, E., McGovern, D., Mockler, D., \& Doku, E. A. (2000). Neuropsychological functioning in first episode psychosis: Evidence of specific deficits. Schizophrenia Research, 43, 47-55.

Shonin, E., Van Gordon, W., Slade, K., \& Griffiths, M. D. (2013). Mindfulness and other Buddhist-derived interventions in correctional settings: A systematic review. Aggression and Violent Behavior, 18, 365-372.

Shonin, E., Van Gordon, W., \& Griffiths, M. D. (2013a). Buddhist philosophy for the treatment of problem gambling. Journal of Behavioral Addictions, doi: 10.1556/JBA.2.2013.001.

Shonin, E., Van Gordon, W., \& Griffiths, M. D. (2013b). Do mindfulness-based therapies have a role in the treatment of psychosis? Australia and New Zealand Journal of Psychiatry, doi:10.1177/0004867413512688.

Shonin, E., Van Gordon, W., \& Griffiths, M. D. (2013c). Mindfulness-based interventions: Towards mindful clinical integration. Frontiers in Psychology, 4, 194, doi: 10.3389/fpsyg.2013.00194. 
Shonin, E., Van Gordon, W., \& Griffiths, M. D. (2013d). Meditation Awareness Training (MAT) for improved psychological wellbeing: A qualitative examination of participant experiences. Journal of Religion and Health, doi: 10.1007/s10943-0139679-0.

Slaghuis, W., \& Curran, C. (2001). Spatial frequency masking positive and negative symptom schizophrenia. Journal of Abnormal Psychology, 110, 488-493.

Turkington, D., Kingdon, D., \& Weiden, P. (2006). Cognitive behavior therapy for schizophrenia. American Journal of Psychiatry, 163, 365-373.

Van Gordon, W., Shonin, E., Sumich, A., Sundin, E., \& Griffiths, M.D. (2013). Meditation Awareness Training (MAT) for psychological wellbeing in a sub-clinical sample of university students: A controlled pilot study. Mindfulness, doi: 10.1007/s12671-0120191-5.

Wardle, H., Moody, A., Spence, S., Orford, J., Volberg, R., Jotangia, D., . . Dobbie, F. (2011). British Gambling Prevalence Survey 2010. London: National Centre for Social Research.

Wells, A. (1997). Cognitive therapy of anxiety disorders: A practice manual and conceptual guide. Chichester: Wiley.

Wilson, W., Diamond, R., \& Factor, R. (1990). Group treatment for individuals with Schizophrenia. Community Mental Health Journal, 26, 361-372.

Won Kim, S., Grant, J. E., Potenza, M. N., Blanco, C., \& Hollander, E. (2009). The gambling symptom assessment scale (G-SAS): A reliability and validity study. Psychiatry Research, 166, 76-84. 
Zubin, J. \& Spring, B. (1977). Vulnerability: A new view on schizophrenia. Journal of Abnormal Psychology, 86, 103-126.
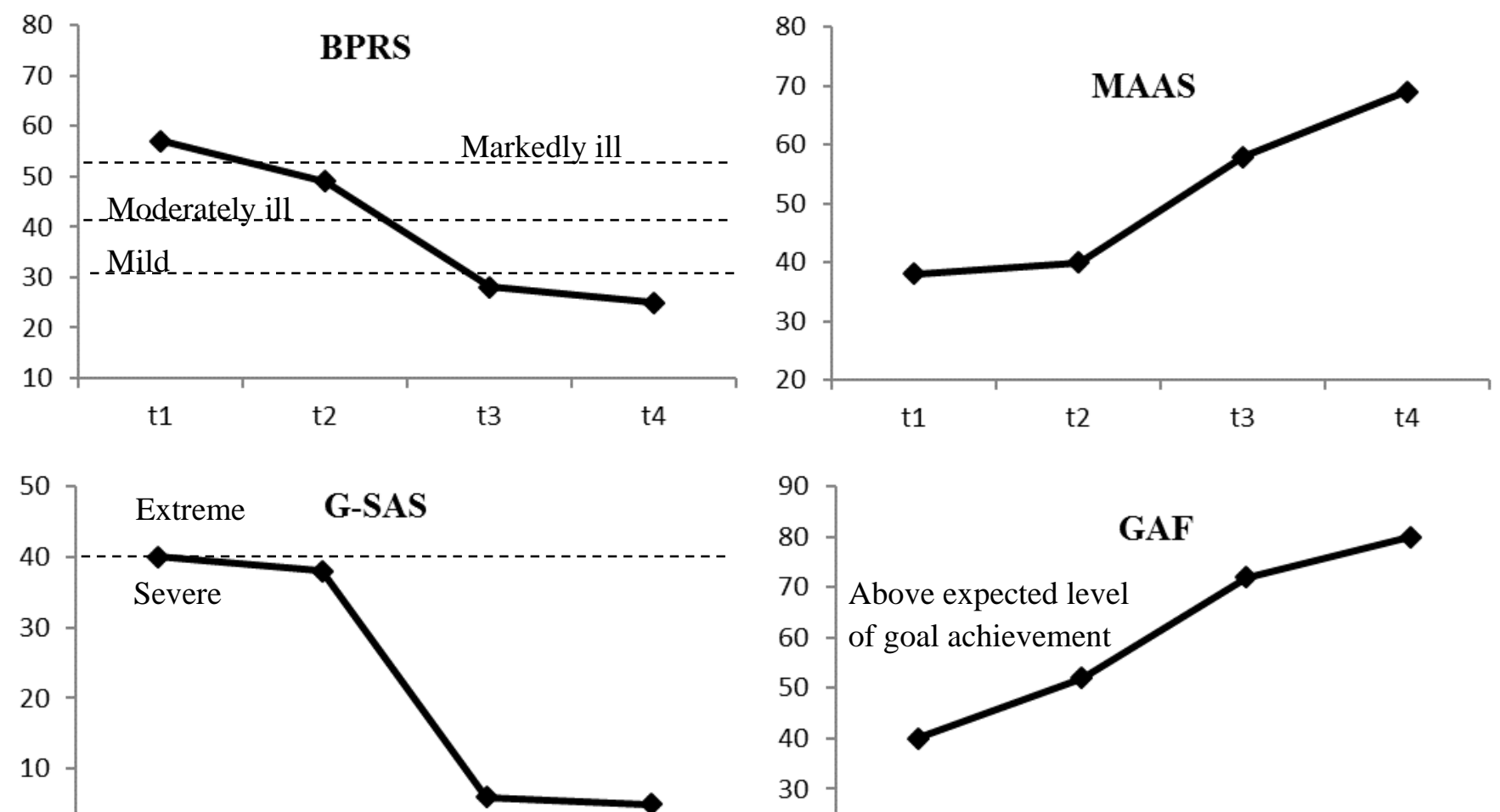
Figure 1 . Change in outcome variable scores over time. $t 1=$ baseline, $t 2=$ week 10 [end of CBT phase $/$ start of MAT phase], $t 3=$ week 20 (therapy termination), $t 4=3$-month followup. 